\title{
Physical monitoring and advice: absence of evidence is not evidence of absence ${ }^{\dagger}$
} COMMENTARY ON... COCHRANE CORNER

\author{
Katharine Smith
}

\begin{abstract}
SUMMARY
Individuals with severe mental illness have increased rates of physical health problems and reduced life expectancy. As a vulnerable population, they have been identified as needing increased physical health monitoring and treatment. The first of two Cochrane reviews considered here assessed the evidence for the benefit of monitoring but found no studies that could be included. The second reviewed the evidence for provision of general physical healthcare advice. Although the results were suggestive of benefit, the evidence, where available, was of poor quality. These reviews highlight an important area for future research to evaluate the relative health and cost benefits of different types of intervention.
\end{abstract}

\section{DECLARATION OF INTEREST}

None

\section{Clinical setting}

Patients who have both physical and mental health needs have poorer outcomes on both accounts (Thornicroft 2011). For example, those with longterm physical health problems are twice as likely to suffer from depression than the general population (Moussavi 2007) and have poorer outcomes for both their depressive and physical illnesses.

Patients with severe mental illness (SMI) such as schizophrenia or bipolar disorder have increased rates of a wide range of physical health problems and these have significant consequences. Lower rates of exercise, higher rates of obesity and smoking, and poorer diet contribute to increased rates of hypertension, high plasma cholesterol and triglycerides, diabetes and obesity, which are often compounded by the effects of long-term psychiatric medications (Thornicroft 2011). Individuals with SMI have poorer access to physical screening and physical healthcare (De Hert 2011), perhaps partly because of 'diagnostic overshadowing', where clinicians misinterpret physical symptoms as a mental health issue (Thornicroft 2011). These factors, combined with higher rates of suicide, accidental and violent death mean that the life expectancy of people with SMI is 15 to 20 years less than for the general population, and this gap has reduced only modestly over the past 20 years (Wahlbeck 2011).

Despite this evidence, physical health problems in SMI are less effectively detected and treated than those in the general population (Thornicroft 2011). Almost a decade ago, the Department of Health published a commissioning framework (Department of Health 2006) intended to provide best practice guidance on the physical health needs of people with SMI, and this was accompanied by investment in 'spearhead primary care trusts' to implement the services it suggested. More recently, the Royal College of Psychiatrists and the National Institute for Health and Care Excellence (NICE) have published guidance documents on physical health monitoring in mental illness (Royal College of Psychiatrists 2009; NICE 2014a,b). These guidelines, based on the convincing evidence of increased physical health problems in those with SMI, suggest programmes of physical health monitoring and health promotion, but do not refer to evidence to support their effectiveness. The available evidence is investigated in this month's pair of Cochrane reviews (p. 74, this issue).

\section{Results of the reviews}

The first review (Tosh 2014a) searched for all randomised clinical trials involving patients with SMI that compared physical health monitoring with standard care or compared different types of health monitoring. The initial search was carried out in 2009 and updated in 2012. No completed studies were identified, so the review is 'empty' of results (Box 1). Only one ongoing study (Jones 2013) was identified. That investigates the benefit of monitoring the oral and dental health of people
ROUND THE CORNER
Katharine Smith is an honorary consultant psychiatrist at the National Institute for Health Research (NIHR) Oxford cognitive health Clinical Research Facility and Oxford University Department of Psychiatry. She has dual accreditation as a consultant in both general adult and old age psychiatry. Correspondence Dr Katharine Smith, NIHR Oxford cognitive health Clinical Research Facility, Warneford Hospital, Oxford OX3 7JX, UK. Email: katharine.smith@psych.ox.ac.uk

'See p. 74, this issue. 
B0X 1 Empty reviews

An 'empty' Cochrane review raises several issues (Yaffe 2012). The first is often disappointment that no studies can be found in an important area. The second issue is to question whether the parameters for inclusion set by the reviewers were so stringent that they excluded usable data. In this case (Tosh 2014a,b), the parameters set were broad and the authors detailed the studies considered with clear reasons for exclusion. The authors avoided a common pitfall in 'empty reviews' of stating conclusions even when no studies meet the inclusion criteria (Green 2007). Empty Cochrane reviews are important, despite their lack of data. They play a key role in highlighting to researchers, commissioners of research and policy makers important questions that require further study. They also inform clinicians, patients and carers when there is lack of robust evidence in favour of (or against) a healthcare intervention (Green 2007).

with SMI, but it is still in protocol format only (planning to report in 2015).

The second Cochrane review searched (in 2009 and 2012) for randomised clinical trials comparing the effects of routine general physical health advice with those of treatment as usual in patients with SMI (Tosh 2014b). The review focused on general advice and excluded a small number of studies of targeted advice interventions (e.g. for obesity or diabetes). In this case, seven studies (1113 patients overall) were identified, but the review's findings were severely limited by the quality of the included trials: the evidence was judged to be of low or very low quality according to the GRADE approach (Box 2). In many of the studies it was unclear whether randomisation and masking ('blinding') had been performed appropriately, details of standard care were not provided and sample sizes were small. In addition, not all the outcome data

\section{BOX 2 GRADE quality assessment}

The Grading of Recommendations Assessment, Development and Evaluation (GRADE) approach (www.gradeworkinggroup.org), used by the Cochrane collaboration, NICE and publications such as the BMJ, assesses the quality of evidence according to the type and quality of the included trials. 'Low quality' indicates that further research is very likely to have an important impact on the confidence in the estimate of effect and is likely to change the estimate. 'Very low quality' indicates that reviewers are very uncertain about the estimate (Higgins 2011: section 12.2.1) could be used, as raw scores were not presented in all cases. At 32\%, the overall attrition rate was high but similar for both groups.

There was some limited evidence that general physical healthcare advice can improve healthrelated quality of life, particularly when assessed using the mental component of the Quality of Life Medical Outcomes Scale (between-group mean difference $\mathrm{MD}=3.70 ; 95 \%$ CI 1.76-5.64; 2 trials, $n=487$ ). However, although this was statistically significant, it was not clear from the original study whether the difference was clinically significant to patients and carers. There was no significant difference between groups on rates of death, where this was reported (relative risk $\mathrm{RR}=0.98,95 \% \mathrm{CI}$ $0.27-3.56$; 2 trials, $n=487$ ), but numbers were too small to show a significant difference in an uncommon event during the study period. The effect on service use had limited evidence from one trial, where at 12-month follow-up, the average uptake of preventive services more than doubled in the intervention group, but remained constant in the usual care group $(\mathrm{MD}=36.90,95 \% \mathrm{CI} 33.07-40.73$; 1 trial, $n=363$ ). In another small trial, significantly more people who received physical health advice attended primary care appointments than those receiving standard care alone $(\mathrm{RR}=1.77,95 \% \mathrm{CI}$ $1.09-2.85 ; 1$ trial, $n=80)$. In summary, although the results were suggestive of some benefit (and showed no harm), the poor quality and small size of the included trials make the findings equivocal.

\section{Conclusions}

Even though no relevant high-quality randomised trials about physical health advice and monitoring for people with SMI have yet been completed, there is increasing pressure for services to take responsibility for providing these interventions to patients. The NICE guidelines for bipolar disorder and for schizophrenia (NICE 2014a,b) recommend regular physical monitoring and advice, and this has significant resource implications of both time and expertise (Box 3). Monitoring should already be performed routinely in primary care, but adherence to an annual physical check of patients with SMI may be as low as 32\% (Yeomans 2014). However, the use of a computerised template has been shown to improve the volume and quality of cardiovascular screening performed (Yeomans 2014). In secondary care, improving physical healthcare to reduce premature mortality in people with SMI is one of the Commissioning for Quality and Innovation (CQUIN) mental health goals for 2014-2015. The Royal College of Psychiatrists has been commissioned by NHS England to 
BOX 3 NICE guideline recommendations for physical monitoring in bipolar disorder and schizophrenia

In addition to specific monitoring recommended for long-term psychotropic medication such as lithium or antipsychotics, NICE recommends an annual check of:

- weight and/or body mass index (BMI)

- diet

- nutritional status

- level of physical activity

- cardiovascular status (including pulse and blood pressure)

- metabolic status, including fasting blood glucose glycosylated haemoglobin $\left(\mathrm{HbA}_{1 \mathrm{c}}\right)$, blood lipid profile

- liver function.

Clinicians should identify at the earliest opportunity patients who have hypertension, abnormal lipid levels, obesity/risk of obesity, diabetes/risk of diabetes, or are physically inactive, and follow the relevant NICE guidance to manage their physical health problems. All patients should be offered a combined healthy eating and physical activity programme

(NICE 2014a,b)

support in-patient service providers in improving the assessment, treatment and communication between clinicians regarding cardiometabolic risk factors. The College suggests the use of a template such as the Lester UK Adaptation of the Positive Cardiometabolic Health Resource and the CQUIN toolkit developed by Rethink Mental Illness (for both see www.rcpsych.ac.uk/workinpsychiatry/ qualityimprovement/cquin.aspx) to improve the physical monitoring and care of in-patients with SMI, and in time to extend this to out-patient care.

Physical healthcare needs can only be addressed once detected and therefore monitoring and advice should be of benefit. However, without evidence from well-conducted research it is difficult to standardise this approach and evaluate the relative health and cost benefits of different types of intervention. Both Cochrane reviews considered here have been updated recently, with no new evidence added. However, by showing the lack of good-quality evidence, these reviews highlight significant gaps and it will be interesting to see how the evidence base develops over the next few years.

\section{Acknowledgements}

The author acknowledges support from the NIHR Oxford cognitive health Clinical Research Facility and thanks John Geddes, Professor of Epidemiological Psychiatry and Andrea Cipriani, Associate Professor at Oxford University Department of Psychiatry for their comments and suggestions. The views expressed are those of the author and not necessarily those of the NHS, the NIHR or the Department of Health.

\section{References}

De Hert M, Correll CU, Moller H-J, et al (2011) Physical illness in patients with severe mental disorders. I. Prevalence, impact of medications and disparities in health care. World Psychiatry, 10: 52-77.

Department of Health (2006) Choosing Health: Supporting the Physical Needs of People with Severe Mental IIIness (Commissioning Framework). Department of Health.

Green S, Higgins JPT, Schunemann HJ, et al (2007) Response to paper by Lang A, Edwards N, and Fleiszer A. Journal of Clinical Epidemiology, 60: $598-9$

Higgins JPT, Green S (eds) (2011) Cochrane Handbook for Systematic Reviews of Interventions (Version 5.1.0). Cochrane Collaboration (http:// handbook.cochrane.org).

Jones HF, Adams CE, Clifton A, et al (2013) An oral health intervention for people with serious mental illness (Three Shires Early Intervention Dental Trial): study protocol for a randomised controlled trial. Trials, 14: 158

Moussavi S, Chatterji S, Verdes E, et al (2007) Depression, chronic disease, and decrements in health: results from the World Health Surveys. Lancet, 370: 851-8.

National Institute for Health and Care Excellence (2014a) Psychosis and Schizophrenia in Adults: Treatment and Management (NICE Clinical Guideline CG178). NICE.

National Institute for Health and Care Excellence (2014b) Bipolar Disorder: The Assessment and Management of Bipolar Disorder in Adults, Children and Young People in Primary and Secondary Care (NICE Clinical Guideline CG185). NICE.

Royal College of Psychiatrists (2009) Physical Health in Mental Health: Final Report of a Scoping Group (Occasional Paper OP67). Royal College of Psychiatrists.

Thornicroft G (2011) Physical health disparities and mental illness: the scandal of premature mortality. British Journal of Psychiatry, 199: 441-2.

Tosh G, Clifton AV, Xia J, et al (2014a) Physical health care monitoring for people with serious mental illness. Cochrane Database of Systematic Reviews, 1: doi 10.1002/14651858.CD008298.pub3.

Tosh G, Clifton AV, Xia J, et al (2014b) General physical health advice for people with serious mental illness. Cochrane Database of Systematic Reviews, 3: doi 10.1002/14651858.CD008567.pub3.

Wahlbeck K, Westman J, Nordentoft M, et al (2011) Outcomes of Nordic mental health systems: life expectancy of patients with mental disorders. British Journal of Psychiatry, 199: 453-8.

Yaffe J, Montgomery P, Hopewell S, et al (2012) Empty reviews: a description and consideration of Cochrane Systematic Reviews with no included studies. PLOS ONE 7(5): e36626.

Yeomans D, Dale K, Beedle K (2014) Systematic computerised cardiovascular health screening for people with severe mental illness. Psychiatric Bulletin, 38: 280-4. 